\title{
Characterization of graphomotor functions in individuals with Parkinson's disease and essential tremor
}

\author{
Nan-Ying $\mathrm{Yu}^{1}$ • Arend W. A. Van Gemmert ${ }^{2} \cdot$ Shao-Hsia Chang ${ }^{1}$
}

Published online: 20 June 2016

(C) Psychonomic Society, Inc. 2016

\begin{abstract}
In this study, we explored the relationship between the clinical features and motor impairments related to the graphomotor function of individuals with Parkinson's disease (PD) and essential tremor (ET). We recruited 46 participants: 12 with PD, 13 with ET, and 21 controls. All participants were asked to perform six graphomotor tasks on a digitizer tablet: drawing straight lines, cursive-connected loops, discrete circles, and continuous circles, and making goal-aimed movements with a stylus in two different directions with three different accuracy constraints. The results showed that although participants with PD were able to draw straight lines slightly faster than controls, they produced cursive-connected loops much slower than controls. In addition, in contrast to controls and individuals with ET, PDs also drew the cursive loops progressively smaller. In the aiming task, we found that equivalent movements with high accuracy constraints were drawn slower by individuals with ET or PD than by controls. However, when performing the equivalent movements with moderate or low accuracy constraints, PDs performed similarly to controls. In contrast to the equivalent movements, PD and ET participants both performed nonequivalent
\end{abstract}

Nan-Ying Yu

ying@mail.isu.edu.tw

Arend W. A. Van Gemmert

gemmert@1su.edu

Shao-Hsia Chang

stacey12@mail.isu.edu.tw

1 Department of Occupational Therapy, I-Shou University, No. 8, Yida Road, Yanchao District, Kaohsiung City 82445, Taiwan

2 School of Kinesiology, Louisiana State University, Baton Rouge, LA 70803, USA movements slower than controls, no matter the demands arising from the accuracy constraints. The present study shows that simple graphic tasks can differentiate impairments in fine motor function resulting from ET and PD.

Keywords Parkinson's disease $\cdot$ Essential tremor $\cdot$ Fine motor function - Graphomotor function · Computerized handwriting evaluation

Bradykinesia, rigidity, resting tremor, and postural instability are the cardinal symptoms of Parkinson's disease (PD). Other symptoms affecting movement control that are associated with PD include shortened movement amplitudes, delays in movement initiation, and high variability of movement execution (Brown \& Marsden, 1991; Flash, Inzelberg, Schechtman, \& Korczyn, 1992; Sheridan, Flowers, \& Hurrell, 1987). Essential tremor (ET) is, like PD, a progressive neurological disease. ET is among the most prevalent movement disorders (Louis, Ottman, \& Hauser, 1998; Thanvi, Lo, \& Robinson, 2006). The characteristic clinical feature of ET is a kinetic tremor (i.e., a tremor that occurs during voluntary movements) of the arms, in contrast to a tremor at rest (Louis, 2005).

The recent literature suggests that ET as a phenotypically heterogeneous degenerative disorder, possibly a comorbid affliction presented with other neurological diseases, including PD, Alzheimer disease, and dystonia (Jankovic, 2002). In ET, rigidity, bradykinesia, and postural instability may accompany postural tremor (Benito-Leon \& Louis, 2006), which symptoms may add to the challenges with producing movements smoothly that arise from the tremor. Thus, kinetic tremor may not only affect the execution of movements among individuals with ET, it may also be present in individuals with PD in their early stages (Duval, Sadikot, \& Panisset, 2004). Although kinetic tremor in individuals with PD has been observed 
during slow alternating movements, it did not seem to affect the accuracy of the movements, and thus it is not clear how it would impact voluntary movements (Duval et al., 2004). It is believed that the tremor cycle might influence the onset of the movements by disturbing the timing of voluntary muscle contractions (Wierzbicka, Staude, Wolf, \& Dengler, 1993). In addition, it has also been suggested that tremor may play a role in the slowing of movements observed in PD, therefore constituting a pathophysiological link between tremor and bradykinesia (Volkmann et al., 1996).

Elble, Higgins, and Hughes (1994) compared fast repetitive wrist flexions between healthy controls and participants with ET. They found that the initiation of the movements of participants with ET was time-locked to the tremor circle, suggesting that not the execution itself, but the movement initiation is affected when performing rhythmic behavior. Support for this suggestion that the execution of rhythmic behavior is unaffected by ET has been provided by Ozekmekci et al. (2005), who found that individuals with ET and controls did not perform differently a sequential-tapping task. Whereas the previous research suggested that initiation is largely unaffected in ET, other studies did show that both movement initiation and execution were affected (Montgomery, Baker, Lyons, \& Koller, 2000; Jimenez-Jimenez et al., 2010). For example, Montgomery and colleagues showed that the reaction time and movement velocity of a wrist flexion and extension task were slower for individuals with ET and PD. Also, JimenezJimenez and colleagues showed that individuals with ET exhibited impairments in rapid repetitive finger movements and visual reaction time tasks (Jimenez-Jimenez et al., 2010), whereas Farkas, Szirmai, and Kamondi (2006) showed higher variability in individuals with ET than in controls when they rhythmically tapped their fingers and made alternating hand movements (Farkas et al., 2006). In general, it can be concluded that individuals with ET have a measurable disability with time-based upper-extremity function (Heroux, Parisi, Larocerie-Salgado, \& Norman, 2006). All of these studies combined show that individuals with ET move slower, and their initiation of movements seems to be affected by the tremor, especially in tasks with time constraints, resulting in some studies not showing impairments because the tremor and the initiation of the rhythmic movements are possibly synchronized. The latter suggestion, together with the paucity of studies addressing how kinetic tremor affects fine motor tasks with relatively high accuracy constraints and lower timing constraints, in part motivated the present study.

Poon, Robichaud, Corcos, Goldman, and Vaillancourt (2011) combined joint movement and force variability measures and found that movement deceleration was more impaired in patients with PD than in those with ET, whereas individuals with ET showed more variable force production than individuals with PD. Therefore, Poon et al. suggested that force variability and peak deceleration can be used as measures to discriminate between PD and ET.

Although the clinical features of PD and ET have been studied extensively, deficits in movement control that result from these features remain largely unknown. To date, uncertainty exists as to whether PD and ET patients have similar degrees of impairment during motor tasks (Duval, Sadikot, \& Panisset, 2006; Montgomery et al., 2000). Therefore, identifying measures indicating movement control proficiency that can differentiate ET from PD is highly desirable, because the prognosis and early treatment options are considerable different for these diseases.

Handwriting and drawing tasks are easy to observe and are functionally important tasks; therefore, these tasks have been used extensively to assess how fine motor function changes as a result of disease and/or medical intervention, even though research using these tasks to investigate the effects of tremor on fine motor tasks has been relatively sparse. For example, Teulings, Contreras-Vidal, Stelmach, and Adler (1997) suggested that the deterioration of fine motor task performance as result of $\mathrm{PD}$ may be caused by a reduction in the capacity to coordinate the timing of the fingers and wrist proficiently, together with an overall reduction in the efficiency to control wrist flexion. Furthermore, Dounskaia, Van Gemmert, Leis, and Stelmach (2009) proposed that PD causes deficits in fine motor control characterized by a reduction of dexterous involvement when producing the variety of shapes necessary to write cursive letters. In the present study, we examined whether graphomotor task performance measures can be used to differentiate between ET and PD.

To verify whether individuals with PD and ET were able to perform the graphomotor tasks, and to determine whether the measures obtained using these tasks would be useful to distinguish these two patient groups, we conducted a pilot study (Yu \& Chang, 2013) in which some individuals participated who would also participate in the present study. In our pilot study, we asked groups of individuals with ET or PD, as well as control older adults, to draw cursive-connected loops and circles and to perform a graphical-aiming task. The results of this pilot showed that individuals with ET made smaller circles and cursive-connected loops than did controls, and that they also made slower movements when drawing circles and cursive-connected loops and when aiming at a target. However, the size reductions and slowness of movements were not as pronounced as the slowness and reductions in size found for individuals with PD in these tasks. In an effort to be more comprehensive, we expanded the number of tasks in the present study by adding straight lines and adding a direction perpendicular to the one used in the original aiming task in the pilot study. These added tasks enabled us to enhance our understanding of mechanisms that may possibly contribute to the movement performance impairments observed in individuals with PD and ET, and ultimately to improve the ability to 
distinguish these two patient groups. We hypothesized that the performance of individuals with PD would differs from the performance of both controls and individuals with ET, because PD affects size control, especially when the movement patterns of the wrist and fingers are more complex (i.e., nonequivalent). Furthermore, we expected the performance deterioration to become more pronounced for individuals with ET when timing constraints aggravated the impact of the kinetic tremor. Finally, we predicted that when accuracy and time constraints were equally important, performance deterioration relative to controls would be shown in both patient groups.

\section{Method}

\section{Participants}

Twelve individuals with PD (mean age $68.75 \pm 3.65$ years), 13 individuals with ET (mean age $67.64 \pm 4.39$ years), and 21 control older adults (mean age $69.15 \pm 4.21$ years) with no history of neurological or current physical disease were recruited for the study (see Table 1 for the characteristics of the individuals with PD and ET). The participants underwent a complete physical and neurological evaluation by a neurologist before the experiment was started. The inclusion criteria for the PD participants in this study were a clinical diagnosis of PD, with initial symptoms including an asymmetric rest tremor of one of the upper limbs. The inclusion criteria for the participants with ET were a diagnosis of definite or probable ET, according to the Washington Heights-Inwood Genetic Study of ET (WHIGET) diagnostic protocol (Louis, Ford, Lee, Andrews, \& Cameron, 1998). Individuals were excluded if the tremor was potentially occurring due to other causes than PD or ET (e.g., psychogenic tremor, neuropathic tremor, or the use of medication known to be associated with drug-induced tremors and/or Parkinsonism). The PD and ET participants were all tested at a medication minimum following overnight withdrawal. The study was approved by I-Shou University's Institutional Review Board overseeing the use of human subjects in research. All participants provided written informed consent prior to participation.

\section{Instruments and apparatus}

Participants were seated on a chair in front of a table on which a digitizer tablet $(487 \times 318 \times 12 \mathrm{~mm}$; Wacom Intuos5, Japan $)$ was positioned in such a way that the tablet's lower edge lined up with the edge of the table. On the digitizer tablet, an A4sized paper was positioned with the vertical and horizontal edges parallel to the horizontal and vertical edges of the digitizer. The participant's forearm was positioned at an angle of about 30 deg with the horizontal edge of the digitizer tablet. A normal-looking wireless electronic pen with force-sensitive tip
(2,048 levels) was used to collect movement data on the digitizer tablet. The axial pen force and the $X$ (horizontal) and $Y$ (vertical) positions of the pen tip were sampled at a frequency of $200 \mathrm{~Hz}$, with a spatial resolution of $0.005 \mathrm{~mm}$. The tablet records the position of the tip of the pen up to a height of $10 \mathrm{~mm}$ when the pen is lifted up from the surface of the digitizer tablet - that is, both movement on the digitizer and air movements above the digitizer were recorded. The electronic pen had a weight and size that is typical for regular pens (length $=$ $150 \mathrm{~mm}$, barrel circumference $=35 \mathrm{~mm}$, weight $=11 \mathrm{~g}$ ).

\section{Procedures}

Graphic tasks The experiment included four separate drawing tasks to assess fine motor function. These tasks were chosen after analysis of the tasks used in a pilot study (Yu \& Chang, 2013). Several participants (eight PD, ten ET, and 13 control participants) had also participated 3 months before in the pilot study. The four graphic tasks were (1) drawing three 50-mm squared crosses (i.e., the horizontal line for nonequivalent movements, and the vertical line for equivalent movements), (2) writing 20-mm tall connected cursive 1ll1lllls (i.e., connected loops with a progression to the right), (3) a single circle with a radius of $20 \mathrm{~mm}$, and (4) ten continuously overlapping circles with a radius of $20 \mathrm{~mm}$. Before every test, the examiner showed and instructed participants how to copy the template. During the trial, a template with the exact dimensions required was presented on the table just above the digitizer tablet. Although the participants were instructed to copy exactly the provided templates, size accuracy was not emphasized in these conditions. The order of task presentation was randomized across participants.

The first two tasks were used to compare the movement velocities per stroke across groups, and to investigate movement velocity changes due to curvature. The connected cursive loops were also used to assess the participants' ability to produce the same-sized template and whether they would maintain size across the eight loops. The latter question was assessed by comparing the size difference between the first and last loops. The two circle-drawing tasks were used to test accuracy and consistency. In addition to performing the latter two tasks with an inking pen, the participants were also required to perform the two circle-drawing tasks with a pen with a noninking filling, thus eliminating any feedback individuals would obtain from the trace of the pen. This manipulation was added to determine whether the visual feedback provided by the trace of the pen benefits the accuracy and consistency of performance when drawing circles.

Graphic aiming tasks In addition to the conditions assessing fine motor control of graphic tasks during a 
Table 1 Patient characteristics

\begin{tabular}{|c|c|c|c|c|c|c|}
\hline Diagnosis & Age & Gender & $\begin{array}{l}\text { Dominant } \\
\text { Hand }\end{array}$ & $\begin{array}{l}\text { Disease } \\
\text { Duration } \\
\text { (years) }\end{array}$ & $\begin{array}{l}\text { Diagnosis of ET/ Motor } \\
\text { UPDRS Scores }\end{array}$ & Medication \\
\hline $\mathrm{ET}$ & 72 & M & $\mathrm{R}$ & 5 & Definite ET & Propanolol \\
\hline ET & 67 & M & $\mathrm{R}$ & 7 & Definite ET & Propanolol \\
\hline ET & 61 & M & $\mathrm{R}$ & 4 & Probable ET & None \\
\hline ET & 72 & M & $\mathrm{L}$ & 4 & Definite ET & Propanolol \\
\hline ET & 72 & $\mathrm{~F}$ & $\mathrm{R}$ & 5 & Definite ET & Propanolol \\
\hline ET & 62 & M & $\mathrm{R}$ & 8 & Definite ET & Propanolol \\
\hline ET & 72 & M & $\mathrm{R}$ & 12 & Definite ET & None \\
\hline ET & 67 & $\mathrm{~F}$ & $\mathrm{~L}$ & 5 & Probable ET & None \\
\hline ET & 63 & M & $\mathrm{R}$ & 4 & Probable ET & None \\
\hline ET & 65 & M & $\mathrm{R}$ & 3 & Probable ET & Propanolol \\
\hline $\mathrm{ET}$ & 71 & $\mathrm{~F}$ & $\mathrm{R}$ & 6 & Definite ET & Propanolol \\
\hline PD & 72 & M & $\mathrm{R}$ & 7 & 32 & Levodopa \\
\hline PD & 69 & $\mathrm{~F}$ & $\mathrm{R}$ & 5 & 31 & Amantadine, \\
\hline PD & 68 & M & $\mathrm{R}$ & 4 & 32 & Amantadine, \\
\hline PD & 63 & M & $\mathrm{R}$ & 6 & 26 & Selegiline \\
\hline PD & 76 & M & $\mathrm{R}$ & 14 & 28 & Levodopa \\
\hline PD & 72 & $\mathrm{~F}$ & $\mathrm{~L}$ & 8 & 32 & Pramipexole \\
\hline PD & 64 & M & $\mathrm{R}$ & 4 & 25.5 & Amantadine \\
\hline PD & 68 & M & $\mathrm{R}$ & 3 & 22 & Pramipexole \\
\hline PD & 67 & M & $\mathrm{R}$ & 2 & 17.5 & Amantadine \\
\hline PD & 66 & M & $\mathrm{L}$ & 3 & 13 & Selegiline \\
\hline PD & 71 & $\mathrm{~F}$ & $\mathrm{R}$ & 6 & 28 & Levodopa \\
\hline PD & 69 & M & $\mathrm{R}$ & 5 & 22 & Pramipexole \\
\hline
\end{tabular}

ET $=$ essential tremor, UPDRS = Unified Parkinson's Disease Rating Scale, $\mathrm{PD}=$ Parkinson's disease, $\mathrm{F}=$ female, $\mathrm{M}=\mathrm{male}, \mathrm{R}=$ right, $\mathrm{L}=$ left

freehand performance, we used a variation of the classical Fitts's (1954) task to test the participants' ability to precisely control the fingers and wrist to cope with amplitude and endpoint accuracy task constraints. Participants were required to make repetitive aiming movements with the pen to target circles that were $25 \mathrm{~mm}$ apart from each other. The accuracy constraint was varied by using target circles with diameters of $2.2,4.4$, and $8.8 \mathrm{~mm}$. Thus, the indexes of difficulty of the three accuracy conditions were $4.51,3.51$, and 2.51 , respectively. These conditions were presented in a random order to participants. In addition to the variation in task difficulty, two different directions were used: The first direction required back-and-forth movements between targets positioned to the upper right and lower left, thus requiring the participant to predominantly make wrist movements, whereas the second direction condition required the participants to move back and forth between targets positioned to the upper left and lower right, thus requiring them to predominantly make finger movements. During a trial, two target circles in one of the diagonal directions were presented.

\section{Data processing and analyses}

Movement velocity The recorded $X$ and $Y$ position data of the pen tip were smoothed with a fourth-order Butterworth lowpass filter with a cutoff frequency of $15 \mathrm{~Hz}$. The local maxima and minima of the $Y$-coordinates were used to segment the 11111111 and aiming task into strokes. The velocity measures were estimated from the sampled data in these ways:

1. Mean velocity: This was determined by averaging the tangential velocity per stroke. The mean velocity per condition for each participant was determined by averaging the values across all trials per condition.

2. Peak velocity: The maximum value of the tangential velocity per stroke was first determined, after which the mean peak velocity per trial was determined by averaging the values across the strokes. The mean peak velocity per condition was determined for every participant by averaging the values of all trials per condition.

3. Ratio of the deceleration phase (RDP): The time of peak velocity and total movement duration were determined. 
The duration of the deceleration phase can be a function of the total movement duration, and therefore the duration of the deceleration phase needed to be normalized for the total movement duration. The normalization procedure resulted in a calculation for the RDP (i.e., [(Total movement duration - Duration till peak velocity of the movement)/Total movement duration] $\times 100 \%$ ). If this ratio is larger than $50 \%$, more time is spent in the deceleration phase - that is, more time is spent breaking the forces exerted to propel the pen toward the target, and thus movement control is less efficient. Efficient movement control occurs when the time spent accelerating (i.e., the forces exerted to propel the pen toward the target) is equal to the time spent decelerating (i.e., the braking forces exerted while the pen approaches the target, so that the pen ends up in the target). Therefore, a bell-shaped velocity profile with a $50 \%$ RDP is assumed to be most efficient.

\section{Accuracy and consistency measures}

Stroke size of the cursive 1 loop The participants were required in the cursive 1-loops condition to copy 111111lls with a height of $20 \mathrm{~mm}$ and a width of $10 \mathrm{~mm}$ per loop. The spatial dimensions of an 1 were determined by averaging the height and width of the two consecutive strokes. To determine the ability to maintain stroke dimensions across a trial, the difference was calculated between the height and width of the first and last ls.

Drawing circles To test the accuracy of area within the curve, the area within the drawn circles was calculated and compared to the area of the template circles. To estimate the spatial accuracy, the mean square error (MSE) of the deviation between the drawn trajectory and the target circle was computed. Figure 1 shows an example of a circle drawn by a participant (solid line) and the reference circle (dotted line). First, the center of the drawn circle was estimated and then after the center was computed, the average radius per sample of the drawn circle was calculated. The center and average radius was used to determine the reference circle. This reference circle was used to calculate the MSE. The MSE was determined by calculating the squared mean of the deviation of the radius per sample between the drawn circle (see Fig. 1, solid line) and the reference circle (see Fig. 1, dotted line).

To assess the consistency of the curvature of each circle, the variation of the average radius (VR) of each circle was computed. The consistency was then determined by taking the coefficient of variation across the ten drawn circles - that is, the standard deviation of the radius across the ten drawn

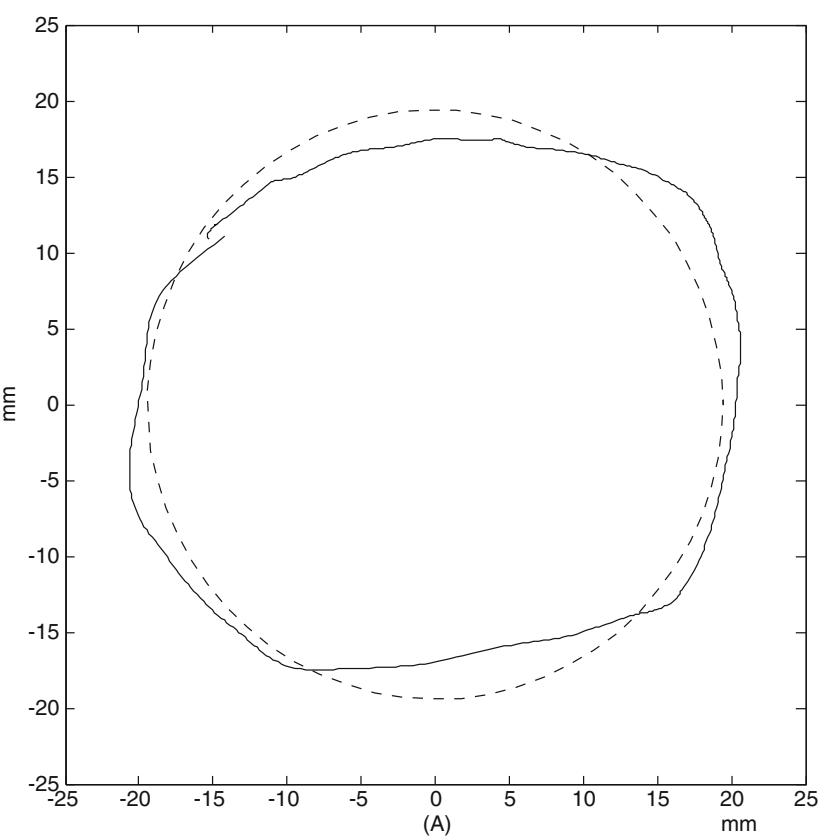

Fig. 1 Example of a circular trajectory (solid line) drawn by a participant. The dotted circle has the same center as the pen movement trajectory

circles was calculated and divided by the mean radius of the ten drawn circles.

\section{Statistical analyses}

The calculated means for all dependent variables per condition per participant were entered in a mixed-factors analysis of variance (ANOVA), with the between-group factor Participant Group (PD, ET, control) and Graphic Condition (crosses, equivalent aiming movements, nonequivalent aiming movements, discrete circles, continuous circles, and loops) as the within-group factor.

To determine how accurately participants were able to copy the template, each drawing's size (height and width) was compared to the template using $t$ tests. Furthermore, to determine consistency when copying the template, a mixed-factors ANOVA with the between-group factor Participant Group (PD, ET, control) and the within-group factor Loop Position (first and last loop) was used to analyze the change across the eight loops among the different groups.

Additionally, the calculated means for all dependent variables per condition per participant were entered in a mixedfactors ANOVA with Participant Group (PD, ET, control) as the between-group factor and Circle Drawing Condition (discrete circle, continuous circle) and Visual Feedback (inking pen, noninking pen) as the within-group factors. This analysis was used to determine whether participant groups were differentially affected by either visual feedback or the circledrawing condition, or by an interaction between the two. Finally, a third mixed-factors ANOVA was used to determine the effects of coordination and accuracy constraints on groups. 
This mixed-factors ANOVA included Participant Group (PD, ET, control) as the between-group factor and ID $(2.51,3.51$, 4.51) and Direction (equivalent, nonequivalent) as the withingroup factors. Bonferroni-corrected post-hoc tests were utilized to determine the locus of significant effects when a main effect of group and/or an interaction with group proved to be significant. All statistical analyses were conducted using the SPSS statistical software (version 12, 2002).

\section{Results}

\section{Velocity measures}

Tables 2 and 3 show the mean and peak velocities of participants drawing crossed straight lines (predominantly with the wrist and fingers), graphic nonequivalent aiming movements, graphic equivalent aiming movements, single circle, continuous overlapping circles, and cursive 1 loops.

In the comparison of stroke velocities across groups, a significant difference was found in the mean velocities, but not in the peak velocities. The ET group showed a significantly slower mean velocity than the normal controls. In the task effect, the results of the mixed-factors ANOVA showed significantly different mean and peak velocities across tasks. The post-hoc test shows that the horizontal strokes were faster than the vertical ones. The participants drew continuously overlapping circles faster than a single circle, and they drew cursive loops significantly slower than the figures in the other tasks. However, we observed no significant difference between the equivalent and nonequivalent aiming movements.

The results of a mixed-factors ANOVA showed a significant interaction between participant group and task. Relative to the NC and ET groups, PD participants drew straight lines and circles faster, whereas the strokes of the cursive 1 loops were produced more slowly. The ranges of movement velocities between the cursive 1 loops and the other tasks were significantly larger in the PD group than in the healthy control and ET groups.

\section{Accuracy and consistency measures}

Table 4 shows the height and width of the cursive-connected loops drawn by the participants. The sizes of the loops drawn by the participants were compared with the template target sizes (i.e., height $20 \mathrm{~mm}$ and width $10 \mathrm{~mm}$ ). The PD group produced significantly larger heights than were required for both the first and last loops $(t=3.658, p=.004$, and $t=3.907$, $p=.002$, for the first and last loops, respectively). No significant differences were found between the heights in the templates and the drawn loops in the other two groups. The mixed-design ANOVA used to determine the effects of group and across loops failed to show significant main effects on cursive loop width or height for group $(F=0.759, p=.474$, $\eta^{2}=.034 ; F=0.717, p=.494, \eta^{2}=.032$, for width and height, respectively) or loop position $\left(F=3.484, p=.069, \eta^{2}=.075\right.$; $F=0.001, p=.979, \eta^{2}=.001$, again for width and height, respectively). However, the interaction between group and loop position reached significance for cursive loop width $(F$ $\left.=4.049, p=.024, \eta^{2}=.158\right)$ and cursive loop height $(F=$ $4.246, p=.021, \eta^{2}=.185$ ). The post-hoc tests showed that the individuals with PD decreased significantly the vertical size (i.e., the height) of the cursive-connected loops across the eight connected cursive loops $(p=.040)$, whereas their decrease of the horizontal size was only marginally significant $(p$ $=.077$ ). This decrease of vertical size shown in individuals with PD was in contrast to the findings for individuals with ET, who increased significantly the horizontal size of the cursive-connected loops across the eight connected cursive loops $(p=.032)$, and the vertical size was also increased, albeit this effect was only marginally significant $(p=.062)$. Whereas individuals with ET and PD either increased or decreased the width and/or height of the loops across the cursive-connected loops, controls did not change the width nor the height of the loops across the eight cursive-connected loops ( $p>.467$ and $p$ $>.355$ for width and height, respectively).

Table 2 Mean stroke velocity across straight lines, aiming movements, circles, and cursive 1 loops

\begin{tabular}{|c|c|c|c|c|c|c|c|}
\hline & $\begin{array}{l}\text { Cross } \\
\text { (horizontal) }\end{array}$ & $\begin{array}{l}\text { Cross } \\
\text { (vertical) }\end{array}$ & $\begin{array}{l}\text { Aiming Movement } \\
\text { (equivalent) }\end{array}$ & $\begin{array}{l}\text { Aiming Movement } \\
\text { (nonequivalent) }\end{array}$ & $\begin{array}{l}\text { Single } \\
\text { Circle }\end{array}$ & $\begin{array}{l}\text { Continuous } \\
\text { Overlapping Circles }\end{array}$ & $\begin{array}{l}\text { Cursive } \\
\text { Loops }\end{array}$ \\
\hline $\mathrm{C}$ & $5.40(3.08)$ & $4.92(2.62)$ & $4.14(1.61)$ & $3.95(1.47)$ & $2.96(1.67)$ & $4.86(3.97)$ & $2.17(0.88)$ \\
\hline ET & $4.05(2.26)^{\#}$ & $3.67(2.00)$ & $2.33(.96)$ & $2.43(1.11)^{*}$ & $2.34(.99)$ & $2.82(1.64)$ & $2.07(0.88)$ \\
\hline PD & $7.57(3.57)$ & $4.28(3.28)$ & $3.63(1.52)$ & $2.72(.85)^{\Delta}$ & $2.32(1.11)$ & $5.27(4.11)$ & $1.55(0.54)$ \\
\hline \multicolumn{3}{|c|}{ Group effect } & \multicolumn{5}{|c|}{$F(2,43)=3.249, p=.049, \eta^{2}=.13$} \\
\hline \multicolumn{3}{|c|}{ Task effect } & \multicolumn{5}{|c|}{$F(6,258)=20.359, p<.001, \eta^{2}=.32$} \\
\hline \multicolumn{3}{|c|}{ Interaction effect } & \multicolumn{5}{|c|}{$F(12,258)=2.477, p=.004, \eta^{2}=.10$} \\
\hline
\end{tabular}

C, control participants; ET, participants with essential tremor; PD, participants with Parkinson's disease. Significant differences between C vs. ET, C vs. $\mathrm{PD}$, and ET vs. PD are denoted by $*,{ }^{\Delta}$, and \#, respectively 
Table 3 Peak stroke velocity across straight lines, aiming movements, circles and cursive 1 loops

\begin{tabular}{|c|c|c|c|c|c|c|c|}
\hline & $\begin{array}{l}\text { Cross } \\
\text { (horizontal) }\end{array}$ & $\begin{array}{l}\text { Cross } \\
\text { (vertical) }\end{array}$ & $\begin{array}{l}\text { Aiming Movement } \\
\text { (equivalent) }\end{array}$ & $\begin{array}{l}\text { Aiming Movement } \\
\text { (nonequivalent) }\end{array}$ & $\begin{array}{l}\text { Single } \\
\text { Circle }\end{array}$ & $\begin{array}{l}\text { Continuous } \\
\text { Overlapping Circles }\end{array}$ & $\begin{array}{l}\text { Cursive } \\
\text { Loops }\end{array}$ \\
\hline $\mathrm{C}$ & $12.78(6.97)$ & $10.72(5.39)$ & $10.65(4.67)$ & $9.33(3.44)$ & $6.38(3.17)$ & $11.63(7.12)$ & 4.44 (1.74) \\
\hline ET & $10.27(4.19)$ & $9.68(4.91)$ & $7.11(2.36)^{*}$ & $6.39(2.67)^{*}$ & $6.50(1.86)$ & $9.75(4.92)$ & $4.78(1.57)$ \\
\hline $\mathrm{PD}$ & $14.54(6.47)$ & $9.19(4.33)$ & $8.89(1.68)$ & $6.55(1.21)^{\Delta}$ & $6.57(1.48)$ & $15.42(7.07)$ & $3.98(1.13)$ \\
\hline \multicolumn{3}{|c|}{ Group effect } & \multicolumn{5}{|c|}{$F(2,43)=1.508, p=.233, \eta^{2}=.07$} \\
\hline \multicolumn{3}{|c|}{ Task effect } & \multicolumn{5}{|c|}{$F(6,258)=29.013, p<.001, \eta^{2}=.40$} \\
\hline \multicolumn{3}{|c|}{ Interaction effect } & \multicolumn{5}{|c|}{$F(12,258)=2.358, p=.007, \eta^{2}=.10$} \\
\hline
\end{tabular}

C, control participants; ET, participants with essential tremor; PD, participants with Parkinson's disease. Significant differences between C vs. ET and C vs. PD are denoted by ${ }^{*}$ and ${ }^{\star}$, respectively

Table 5 shows the accuracy and consistency measures of the circles drawn with and without an inking pen. Comparing the sizes to the template, we found no significant differences between the two conditions and the three groups (all $t \mathrm{~s}<1.469$ and $p \mathrm{~s}>$.162). Circle sizes were not significantly affected by group or inking condition (i.e., visual feedback condition). However, circle size did show a significant interaction of inking condition with group: In the post-hoc analyses, the PD group drew the circles significantly larger using an inking pen than when drawing them using a noninking pen.

Whereas group did show a significant main effect on $M S E$ s, the difference between the MSEs of the circles drawn with and without an inking pen did not reach significance. The $M S E$ of the PD participants was found to be significantly larger than those of the controls and ET participants. Group and inking condition did not show a significant interaction for MSEs.

Group and inking condition both showed significant main effects on VRs. The VR increased when participants used a noninking instead of an inking pen. Furthermore, inking condition interacted significantly with group for VRs: Without ink, the PD group showed a larger VR than the other two groups.

\section{Graphic aiming tasks}

Table 6 shows the mean velocities of participants performing the aiming movements. The mixed-factors ANOVA showed that movement velocities differed significantly across

Table 4 Comparison of height and width of cursive 1 loops

\begin{tabular}{|c|c|c|c|c|}
\hline & \multicolumn{2}{|l|}{ Height (mm) } & \multicolumn{2}{|l|}{ Width (mm) } \\
\hline & First Loop & Last Loop & First Loop & Last Loop \\
\hline $\mathrm{C}$ & $20.8(11.9)$ & $21.9(14.2)$ & $8.8(4.8)$ & $9.3(5.0)$ \\
\hline ET & $23.0(11.0)$ & $25.6(17.8)$ & $9.7(4.1)$ & $11.8(4.6)^{*}$ \\
\hline PD & $27.7(7.3)$ & $23.9(3.4)^{*}$ & $10.0(1.5)$ & $9.4(1.6)$ \\
\hline
\end{tabular}

${ }^{*}$ Significant difference between the first and last loops participant groups and task difficulties, whereas group did not interact significantly with task difficulty. From the Bonferroni-corrected post-hoc tests, the ET and PD groups showed significantly slower movements than the NC group ( $p \mathrm{~s}=.003$ and .026 , respectively). The mean velocity of the aiming movements was significantly higher when aiming at the large than at the medium circle, which aiming movement velocity in turn was higher than the one for aiming at the small circle (both $p \mathrm{~s}<.001$ ).

The mixed-factors ANOVA showed that the RDPs differed significantly across participant groups and task difficulties (for the statistics, see Table 6). Furthermore, group interacted significantly with task difficulty for RDPs (see Table 6). Bonferroni-corrected post-hoc tests revealed that the PD group exhibited the largest RDP, as compared to the other two groups (both $p s<.001$ ). In addition, the ET group exhibited a significant larger RDP than did the control group $(p<$ .001). The RDPs across the task difficulties proved to differ significantly when aiming at the small circle was compared to aiming at a large circle $(p=.016)$. Further analysis revealed that RDP differed significantly across task difficulties in the healthy controls $\left(F=4.664, p=.015, \eta^{2}=.189\right)$ and in individuals with $\mathrm{ET}\left(F=12.856, p<.001, \eta^{2}=.517\right)$, whereas the differences in task difficulty did not affect the RDPs of PD participants $\left(F=0.611, p=.552, \eta^{2}=.053\right)$. We showed that the RDPs of both healthy controls and ET participants were significantly higher when they were aiming at the small circles than when they were aiming at the large circles, whereas only the PD group showed a significant higher RDP when aiming movements at the small circles than when aiming at the medium circles. The PD participants did not show a significant difference in RDPs across the aiming tasks.

\section{Discussions}

The results from this study showed that among the three groups, the PD participants made the fastest movements per stroke for straight lines, especially in the horizontal direction, 
Table 5 Size, error (MSE), and variability of the radius (VR) in copying circles

\begin{tabular}{|c|c|c|c|c|c|c|}
\hline & Size $^{\mathrm{a}}$ & Size $^{b}$ & $M S E^{\mathrm{a}}$ & $M S E^{\mathrm{b}}$ & $\mathrm{VR}^{\mathrm{a}}$ & $\mathrm{VR}^{\mathrm{b}}$ \\
\hline $\mathrm{C}$ & $17.53(6.71)$ & $19.55(6.16)$ & $6.5(2.5)$ & $5.8(2.0)$ & $4.8(4.5)$ & $6.5(5.6)$ \\
\hline ET & $16.53(8.61)$ & $17.36(7.24)$ & $7.7(2.4)$ & $7.4(2.2)$ & $5.8(8.4)$ & $8.5(10.0)$ \\
\hline $\mathrm{PD}$ & $20.73(5.80)$ & $18.53(4.64)$ & $15.5(6.5)$ & $15.1(9.7)$ & $18.4(7.4)$ & $29.0(17.1)$ \\
\hline \multicolumn{7}{|l|}{ Main Effects } \\
\hline Group & \multicolumn{2}{|c|}{$F(2,43)=1.387, p=.261, \eta^{2}=.062$} & \multicolumn{2}{|c|}{$F(2,43)=17.527, p<.001, \eta^{2}=.449$} & \multicolumn{2}{|c|}{$F(2,43)=25.767, p<.001, \eta^{2}=.475$} \\
\hline Visual feedback & \multicolumn{2}{|c|}{$F(1.43)=0.375, p=.543, \eta^{2}=.009$} & \multicolumn{2}{|c|}{$F(1.43)=1.333, p=.255, \eta^{2}=.030$} & \multicolumn{2}{|c|}{$F(1.43)=19.416, p<.001, \eta^{2}=.375$} \\
\hline Interaction & \multicolumn{2}{|c|}{$F(2,43)=7.436, p=.002, \eta^{2}=.257$} & \multicolumn{2}{|c|}{$F(2,43)=0.137, p=.873, \eta^{2}=.006$} & \multicolumn{2}{|c|}{$F(2,43)=7.638, p=.001, \eta^{2}=.262$} \\
\hline
\end{tabular}

${ }^{\mathrm{a}}$ Inking condition; ${ }^{\mathrm{b}}$ Noninking condition. C, control participants; ET, participants with essential tremor; PD, participants with Parkinson's disease

which were even faster in individuals with PD than among healthy controls. The ET and PD groups showed significantly larger RDPs than did the NC group. We also found significant differences between the ET and PD groups. The PD group showed a larger RDP than the ET group. The variables that proved to be significant showed medium to large effect sizes, and it should be noted that the effect sizes were large for all variables that distinguished between the groups. This suggests that PD participants have more deficits related to movement slowing than do individuals with ET or healthy controls. As was revealed by Poon et al. (2011), the reduced peak deceleration in individuals with PD significantly differed from the peak decelerations of both of the other groups (i.e., healthy controls and individuals with ET), suggesting that individuals with PD are less able to modulate the deceleration of movements than are individuals with ET, and thus that this variable could be used to distinguish between these two patient groups.

In contrast to simple straight movement, among three groups the PD group showed the slowest cursive l-loop drawing. Their movement velocity reduced intensively in cursive 1loop drawing. A proposed deficit in PD is the reduced ability to recruit sufficient muscle force during the initiation of movement, which has been suggested to result in undershooting of targets and approaching them in several smaller steps instead of one smooth movement (Berardelli, Rothwell, Thompson, $\&$ Hallett, 2001). PD participants were slower when drawing curves than ET participants, which could have been caused by the demands involved when producing a complex and multisegmented movement. The demands required to produce these curves possibly enhanced the force modulation difficulties encountered by PD participants. In agreement with our suggestion are the findings of the study by Poon et al. (2011), which showed that individuals with ET, as compared to individuals with PD, have shorter torque rise times and do not exhibit as much deterioration in force recruitment.

Drawing cursive-connected loops from left to right (i.e., l's) resulted in writing progressively smaller loops in individuals with PD, whereas control and ET individuals did not show a significant progressive reduction of loop sizes when writing the loops along the horizontal axis of the tablet. Therefore, this suggests that whereas individuals with PD are affected by the coordination complexity requirements needed to produce cursive-connected loops, controls and individuals with ET do not seem to be rigorously influenced by these coordination requirements of the writing task. Drawing cursive-connected loops requires a constant readjustment of the coordination

Table 6 Results of accuracy-speed tasks

\begin{tabular}{|c|c|c|c|c|c|c|}
\hline & \multicolumn{3}{|c|}{ Mean Velocity } & \multicolumn{3}{|c|}{ Ratio of the Deceleration Phase (\%) } \\
\hline & \multicolumn{3}{|c|}{ Target Width } & \multicolumn{3}{|c|}{ Target Width } \\
\hline & Large & Medium & Small & Large & Medium & Small \\
\hline $\mathrm{C}$ & $4.53(1.73)$ & $3.89(1.45)^{*}$ & $3.44(1.43)^{* \Delta}$ & $43.6(6.6)$ & $45.7(5.0)$ & $47.5(5.7)^{\Delta}$ \\
\hline ET & $2.78(1.35)$ & $2.42(1.11)^{*}$ & $2.09(0.91)^{* \Delta}$ & $56.3(5.8)$ & $58.3(6.2)$ & $60.5(5.4)^{* \Delta}$ \\
\hline $\mathrm{PD}$ & $3.27(1.57)$ & $2.55(0.58)$ & $2.34(0.57)^{\triangle}$ & $67.5(4.8)$ & $67.1(6.9)$ & $65.9(5.7)$ \\
\hline Group & \multicolumn{3}{|c|}{$F(2,43)=7.284, p=.002, \eta^{2}=.253$} & \multicolumn{3}{|c|}{$F(2,43)=73.14, p<.001, \eta^{2}=.773$} \\
\hline Task difficulty & \multicolumn{3}{|c|}{$F(2,86)=30.514, p<.001, \eta^{2}=.415$} & \multicolumn{3}{|c|}{$F(2,86)=4.043, p=.021, \eta^{2}=.086$} \\
\hline Group $\times$ Task Difficulty & \multicolumn{3}{|c|}{$F(4,86)=0.748, p=.562, \eta^{2}=.034$} & \multicolumn{3}{|c|}{$F(4,86)=2.734, p=.034, \eta^{2}=.113$} \\
\hline
\end{tabular}

C, healthy control participants; ET, participants with essential tremor; PD, participants with Parkinson's disease. Significant differences between the large vs. medium and the medium vs. small tasks are denoted by the symbol $*$. The symbol ${ }^{\triangle}$ denotes significant difference between the large vs. small tasks 
between the wrist and fingers. PD participants showed that they had difficulties when drawing curves, especially when the radius was small, as in the 111111lls task. These tasks may elicit symptoms commonly observed in individuals with PD, such as bradykinesia and micrographia.

Pertaining to the reduced size and speed of handwriting and drawing movements, early studies by Viviani and Terzuolo (1982) observed a systematic relationship between the velocity of the end-effector trajectory and the geometric path that it describes (Viviani \& Terzuolo, 1982). This observation was quantified by Lacquaniti, Terzuolo, and Viviani (1983) as a " $2 / 3$ power law." According to this law, the movement velocity is proportional to the radius of curvature, raised to the power of two thirds. This means that small-sized curves tend to reduce the movement velocity. This may explain the coexisting reduction of movement velocities and stroke sizes in the PD participants. These participants may have had difficulty maintaining the same velocity across the eight loops, resulting in significant reductions in height and width when drawing repetitive loops with a progression to the right.

PD participants did draw the circles with larger MSEs and showed larger variations when drawing consecutive circles than did the control and ET groups. This pattern of findings suggests that kinetic tremor, symptomatic for individuals with ET, does not significantly impair fine motor control when drawing circles. Thus, we suggest that PD participants show these difficulties when drawing circles because they have difficulty coordinating their impaired motor system with a visuospatial function that is still intact. This suggestion is supported by a study by Dounskaia et al. (2009), who showed that PD individuals consistently deformed circles into right-tilted ovals. Other support for our suggestion has come from a study in which individuals with PD were asked to draw a house containing windows and doors (Kulkarni, Lafaver, \& Tarsy, 2013). This study showed that participants with PD often undershoot when drawing, causing doors not to be attached to the bottoms of houses, which created the appearance of a "floating door." Individuals with ET in this study did not show this pattern resulting in a "floating door." The difference between geometric drawings between individuals with PD and individuals not afflicted with PD had, according to the experimenters, a positive predictive value for PD (Kulkarni et al., 2013), because Kulkarni et al. believed that it correlates with hypometric manual movements, causing the inadequate stroke sizes observed in individuals with PD.

It has been shown that individuals with PD are less consistent when using a noninking pen (significantly larger VR), suggesting that visual feedback of the trace improves their performance, possibly due to the use of the trace, when using an inking pen, as a constraint on the movement, making it less inconsistent. Teulings et al. (1997) also showed that PD participants rely on visual feedback of previous and ongoing strokes to program subsequent strokes. Our results, together with those of Teulings et al., suggest that improving visual cues or feedback in clinical training might improve the training outcomes of individuals with PD.

In the test of accuracy-speed tasks, the ET and PD participants showed different results from the healthy participants. The ET participants showed difficulty in all tasks, but the case was not so obvious among the PD participants. It seems that the difficulty for the ET group was not solely the result of coordination complexity. Previous research has shown that ET participants are slower than healthy individuals at rapid alternating movements (Duval et al., 2006; Montgomery et al., 2000). Trillenberg et al. (2006) found that the hand movements of ET participants take longer, which is accompanied by decreases in peak acceleration, increases in the latency of peak velocity, and a reduction of peak deceleration. Trillenberg et al. argued that these results were compatible with cerebellar dysfunction of the control of hand movements. A previous study (Deuschl, Wenzelburger, Löffler, Raethjen, \& Stolze, 2000) had revealed that both cerebellar and ET participants prolong the total movement duration for goaldirected hand movements. In agreement with this finding, the ET participants in the present study exhibited significantly longer movement times in the aiming test than did healthy controls, which suggests that ET has a cerebellar pathophysiology.

Although no significant interaction of group and task difficulty was found, we still decided to explore possible differences between the groups when task difficulty was varied, because visual inspection suggested that an interaction effect might have been occluded due to the large variances within groups. In light of this observation, together with our aim to explore possible differences between groups, it was decided to explore with an additional analysis whether any of the groups showed a tendency to be affected differentially by difficulty. The results showed that the mean velocities in the healthy controls and ET participants were significantly higher when aiming at the large circle than when aiming at the medium circle ( $p \mathrm{~s}=.001$ and .026$)$, whereas the aiming movements to the medium circles were significantly faster than the aiming movements to the small circle ( $p \mathrm{~s}=.018$ and .013 ). The PD participants showed significantly higher mean velocities when aiming at the large circle than when they aimed at the small circle $(p=.048)$. They showed impairment particularly in the tasks with a smaller target size. From the result of the exploratory analysis, it seems that difficulty affected the groups differently. This finding of the exploratory analysis, despite the nonsignificance of the interaction between group and difficulty, suggests that future experiments will need to increase the difference in difficulty between aiming conditions, in addition to possibly increasing the number of participants per group to increase power.

From the results of this study, impaired movement performance is more predictable in PD and ET participants than in 
healthy controls. Furthermore, the slowness and irregularity of the movements of PD and ET patients cannot be fully explained by tremor. Some common pathogenic mechanisms leading to bradykinesia may contribute to these impairments. Unlike conventional clinical evaluation, computerized evaluation provides a much more quantitative approach. The graphomotor tasks employed for studying fine motor control showed the possibility of characterizing the fine motor impairments in PD and ET participants. One clinical implication of our findings is that an evaluation of participants' simple geometric drawings - that is, straight lines and cursive 1 loopsprovides insight into the deficits that affect their drawing performance. Another clinical implication is that individuals with PD show deficits when they have to coordinate their wrist and fingers when required to perform a graphomotor task. A third clinical implication is that the deceleration phases of the aiming movements of the ET and PD groups were prolonged as compared to controls. Furthermore, this parameter differed also between ET and PD groups. It is possible that these features can be used to discriminate between individuals with ET and $\mathrm{PD}$, which may improve early diagnosis and intervention protocols for individuals with ET and PD.

Author note The authors thank the Ministry of Science and Technology of the Republic of China for supporting this work financially under Contract Nos. NSC-100-2221-E-214-041 and NSC-101-2221-E214-005.

\section{References}

Benito-Leon, J., \& Louis, E. D. (2006). Essential tremor: Emerging views of a common disorder. Nature Clinical Practice and Neurology, 2, 666-678.

Berardelli, A., Rothwell, J. C., Thompson, P. D., \& Hallett, M. (2001). Pathophysiology of bradykinesia in Parkinson's disease. Brain, 124, 2131-2146.

Brown, R. G., \& Marsden, C. D. (1991). Dual task-Performance and processing resources in normal subjects and patients with Parkinsons-disease. Brain, 114, 215-231.

Deuschl, G., Wenzelburger, R., Löffler, K., Raethjen, J., \& Stolze, H. (2000). Essential tremor and cerebellar dysfunction clinical and kinematic analysis of intention tremor. Brain, 123, 1568-1580.

Dounskaia, N., Van Gemmert, A. W. A., Leis, B. C., \& Stelmach, G. E. (2009). Biased wrist and finger coordination in Parkinsonian patients during performance of graphical tasks. Neuropsychologia, 47, 2504-2514.

Duval, C., Sadikot, A. F., \& Panisset, M. (2004). The detection of tremor during slow alternating movements performed by patients with early Parkinson's disease. Experimental Brain Research, 154, 395-398.

Duval, C., Sadikot, A. F., \& Panisset, M. (2006). Bradykinesia in patients with essential tremor. Experimental Brain Research, 1115, 213-216.

Elble, R. J., Higgins, C., \& Hughes, L. (1994). Essential tremor entrains rapid voluntary movements. Experimental Neurology, 126, 138143.

Farkas, Z., Szirmai, I., \& Kamondi, A. (2006). Impaired rhythm generation in essential tremor. Movement Disorders, 21, 1196-1199.
Fitts, P. M. (1954). The information capacity of the human motor system in controlling the amplitude of movement. Journal of Experimental Psychology, 47, 381-391. doi:10.1037/h0055392

Flash, T., Inzelberg, R., Schechtman, E., \& Korczyn, A. D. (1992). Kinematic analysis of upper limb trajectories in Parkinsons-disease. Experimental Neurology, 118, 215-226.

Heroux, M. E., Parisi, S. L., Larocerie-Salgado, J., \& Norman, K. E. (2006). Upper extremity disability in essential tremor. Archives of Physical Medicine and Rehabilitation, 87, 661-670.

Jankovic, J. (2002). Essential tremor: A heterogenous disorder. Movement Disorders, 17, 638-644.

Jimenez-Jimenez, F. J., Rubio, L., Alonso-Navarro, H., Calleja, M., Pilode-la-Fuente, B., Plaza-Nieto, J. F., . . Agundez, J. A. (2010). Impairment of rapid repetitive finger movements and visual reaction time in patients with essential tremor. European Journal of Neurology, 17, 152-159.

Kulkarni, O., Lafaver, K., \& Tarsy, D. (2013). The "floating door sign" in Parkinson's disease. Parkinsonism \& Related Disorders, 19, 825-826.

Lacquaniti, F., Terzuolo, C., \& Viviani, P. (1983). The law relating the kinematic and figural aspects of drawing movements. Acta Psychologica, 54, 115-130.

Louis, E. D. (2005). Essential tremor. Lancet Neurology, 4, 100-110.

Louis, E. D., Ford, B., Lee, H., Andrews, H., \& Cameron, G. (1998). Diagnostic criteria for essential tremor-A population perspective. Archives of Neurology, 55, 823-828.

Louis, E. D., Ottman, R., \& Hauser, W. A. (1998). How common is the most common adult movement disorder? Estimates of the prevalence of essential tremor throughout the world. Movement Disorders, 13, 5-10.

Montgomery, E. B., Baker, K. B., Lyons, K., \& Koller, W. C. (2000). Motor initiation and execution in essential tremor and Parkinson's disease. Movement Disorders, 15, 511-515.

Ozekmekci, S., Kiziltan, G., Vural, M., Ertan, S., Apaydin, H., \& Erginoz, E. (2005). Assessment of movement time in patients with essential tremor. Journal of Neurology, 252, 964-967.

Poon, C., Robichaud, J. A., Corcos, D. M., Goldman, J. G., \& Vaillancourt, D. E. (2011). Combined measures of movement and force variability distinguish Parkinson's disease from essential tremor. Clinical Neurophysiology, 122, 2268-2275.

Sheridan, M. R., Flowers, K. A., \& Hurrell, J. (1987). Programming and execution of movement in Parkinsons-disease. Brain, 110, 1247-1271.

Teulings, H. L., Contreras-Vidal, J. L., Stelmach, G. E., \& Adler, C. H. (1997). Parkinsonism reduces coordination of fingers, wrist, and arm in fine motor control. Experimental Neurology, 146, 159-170.

Thanvi, B., Lo, N., \& Robinson, T. (2006). Essential tremor-The most common movement disorder in older people. Age and Ageing, 35, 344-349.

Trillenberg, P., Fuhrer, J., Sprenger, A., Hagenow, A., Kompf, D., Wenzelburger, R., . . . Helmchen, C. (2006). Eye-hand coordination in essential tremor. Movement Disorders, 21, 373-379.

Viviani, P., \& Terzuolo, C. (1982). Trajectory determines movement dynamics. Neuroscience, 7, 431-437.

Volkmann, J., Joliot, M., Mogilner, A., Ioannides, A. A., Lado, F., Fazzini, E.,. . . Llinás, R. (1996). Central motor loop oscillations in Parkinsonian resting tremor revealed by magnetoencephalography. Neurology, 46, 1359-1370.

Wierzbicka, M. M., Staude, G., Wolf, W., \& Dengler, R. (1993). Relationship between tremor and the onset of rapid voluntary contraction in Parkinson's disease. Journal of Neurology, Neurosurgery, and Psychiatry, 56, 782-787.

Yu, N. Y., \& Chang, S. H. (2013). Comparisons of fine motor functions in subjects with Parkinson's disease and Essential tremor. International Journal of Medical, Pharmaceutical Science and Engineering, 7, $398-401$. 